\title{
Development Plan for the Project: Integrated System for Patient Localization, Tracking and Monitoring in an Indoor Environment
}

\author{
Luigi Bibbò* \\ Department of Information Engineering, Infrastructure, and Sustainable Energy, University of Reggio Calabria, Italy
}

\begin{abstract}
Currently, the health care problem for older people is becoming of considerable size and involves the whole civilized world. The number of people in need of assistance is constantly growing due to life extension, and the social cost is becoming unsustainable. Technological evolution and IT development can help contain spending by providing efficient health services and improving the quality of life. This article highlights methodological and technical aspects to support the development of an integrated system for locating and monitoring people in their homes or nursing homes. This system complements the home care service by avoiding hospitalization in health facilities with more significant financial burdens.
\end{abstract}

KEYWORDS: Inertial sensors; Environment sensors; Indoor positioning; Human activity; Convolutional neural networks

\section{INTRODUCTION}

The object is the design of a prototype that can provide an efficient technologic instrument in healthcare assistance through the remote real-time monitoring of patients and their care. The system must be made of devices that enable the caregivers to operate on time and ensure efficient performance in the whole process of the healthcare system. For example, we can design the system with wearable sensors and sensor badges to communicate. In addition, we use a wireless infrastructure and devices for the communication network. Finally, it targets to localize the moving people and monitor their position to provide efficient and practical information to the healthcare system. This network, called WSN (Wireless Sensor Network), is made by slight size sensors node static and mobile that allows the localization and tracking of the patient. In addition, it can monitor their biochemical parameters like blood pressure, heartbeat, oxygen saturation different surrounding environmental parameters [1].

\section{CONTENTS}

The WSN is made by sensors node to gather cooperation, limited processing, and data transmission of monitored patients to the remote center using a wireless channel [2]. To make all this function, the WSN needs a particular node that can wirelessly communicate and be placed in different points within the area to monitor. The components of a WSN are the target node, sensor node, and sink node. These nodes must have the following features: small size, low power, cheap, intelligent, multifunctional, and with wireless interface to communicate each with others. The WSN can be of two types according to the dislocation of sensor nodes: 1) structured network when the nodes are arranged in a predetermined manner, 2) unstructured when many nodes are placed randomly [3]. According to the application, the components of sensor nodes are assembled in a single PCB (Printed Circuit Board) or more PCB.

\section{Characteristics}

A WSN must have these characteristics [4]:

a. Distribution: The nodes can be placed in a not predefined location making the network dynamic.
Quick Response Code:

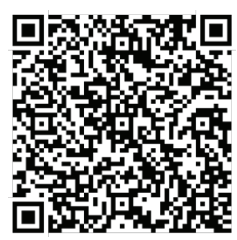

Address for correspondence: Luigi Bibbò, Department of Information Engineering, Infrastructure, and Sustainable Energy, University of Reggio Calabria, Italy

Received: December 31, 2021 Published: January 10, 2022

How to cite this article: Luigi Bibbò, Development Plan for the Project: Integrated System for Patient Localization, Tracking and Monitoring in an Indoor Environment. 2022- 4(1) OAJBS. ID.000375. DOI: $10.38125 / \mathrm{OAJBS} .000375$ 
b. System lifetime: Lifetime depends on a battery. To supply power to the network, we use some shrewdness: secondary power, data aggregation, short transmission range, multi-hop routing.

c. Cooperation: The limited processing ability and memory of sensor nodes require that they interact in a way that there is an efficient processing capability [5].

d. Localization: Since each node interacts with its neighbours, it must know its position and localization of its neighbours to the processing and cooperation. It must be adopted localization technique to identify the places of nodes [6].

\section{Components}

In a WSN, the technology components are:

a. Time synchronization (correct sequencing in the transmission)
b. Localization
c. Data aggregation
d. Power management
e. Security administration

\section{FUNCTIONALITY}

The hypothesized system must support a patient's monitoring system within the healthcare system. It includes two main activities:

\section{a. Localization and tracking of patients}

Correct knowledge of a patient location is essential in cases where it is necessary to assist urgently.

\section{b. Patients monitoring}

It's necessary to know the status of the patients when they move both inside and outside the home and hosting institution. Therefore, we must collect information to check any abnormal monitored parameters (movement characteristics, heartbeat, blood pressure, etc.).

\section{Localization and Tracking of Patients}

The patient is the main subject in the healthcare assistance system. He requires the care at the right moment with a high professional level, wants to participate in its therapeutic plan, and has more information on its disease's progress. These reasons are simply an improvement of the care quality and the target of achieving an excellent outcome. Some patients are cared for in unique health structures by closed monitoring; others require remote monitoring by assistance according to their needs [7].

We consider elder patients affected by Alzheimer's disease and mental disorder in this case. They can run away from their control area with the risk for their health. Then it's necessary to localize, tracking and alert caregivers to avoid serious situations.

Knowing the patient's location is essential because it allows activating the request of necessary healthcare. The localization is the process through which it's possible to identify and determine the coordinates of the sensor nodes or estimate their spatial relationship. It can be both network-centric localization and selflocalization. In network-centric, a central unit is used to estimate the position of the nodes based on information gathered by some reference nodes. In self-localization, the position is calculated via the target node itself.

For the localization of unknown nodes, we must first measure some metrics and then use the algorithm to calculate the position itself.

Measurement techniques: The metrics used are distance, angle, and connectivity [8].

A. Techniques based on the distances between a node and an anchor (known node):

i. TOA (Time of Arrival) is based on the propagation time between a known node and one to localize.

ii. TDOA (Time Difference of Arrival) is based on the difference of time arrival of two signals with different propagation models to the same receiver.

iii. RTF (Roundtrip Time of Flight) is based on sending a signal from a node to a second node and receiving the response at the sending node.

iv. RSS (Received Signal Strength) is based on the use of the indicator RSS to estimate the distance between two nodes considering the intensity of a radio signal decreases with the square of distances from the signal source.

B. Technique based on the angle of reception: AOA It's based on the determination of the angle between the propagation direction of the received signal and one reference predetermined.

C. Technique based on connectivity: Radio hop count. Using RSS as an indicator of connectivity between sensor nodes makes it possible to draw a graph with vertices nodes. The hop count between two nodes can be considered the length of the shortest route between the corresponding vertices nodes.

Positioning technique: The positioning technique computes the location of unknown nodes based on measured metrics and can be [9]:

a) Lateration

b) Multilateration

c) Angulation

a) Lateration: We estimate the node position from the intersection of 3 circles centered at three anchor nodes, whose position is determined by the distance measured by the node to be located. The distance can be resolved using one of the measuring techniques.

b) Multilateration: It is a technique that estimates the node's position more accurately using four circles centered at four neighbour anchor nodes. It uses the statistical method of maximum likelihood estimation (MLE) applied to the intersection area of 4 rings.

c) Angulation: The method estimates the position of a node by using the direction relative to 2 anchors instead of the distance

\section{Localization System: Bluetooth and Deep Learning}

We can use new technologies for localization and tracking of patients: Bluetooth low energy (BLE) and convolutional neural network (CNN) [11]. These technologies are a valid instrument to develop an accurate real-time location system (RTLS) for indoor 
tracking of patients and clinic staff as an alternative to RFID (Radio Frequency Identification). Compared to RFID, Bluetooth is less expensive and doesn't require extensive cabling. The availability of BLE tags and detector make this technology versatile for future implementations, easy setting, and integrates with different hardware [12]. In addition, the Bluetooth sensor network installed in several other areas to oversee enables to acquire and collect extensive positional data to transfer to a convolutional neural network for analysis and classification of location [13].

The evolution of technologies based on Deep Learning (DL) algorithms has proved very effective in indoor localization, estimating spatial and temporal coordinates, and the other information connected with the type of activity. Deep Learning models effectively carry out the ability to classify signals and extract significant characteristics from the acquired data. The structural architecture of the DL models made up of multiple layers gives them a high capacity to learn the descriptive features from complex data, favoring the ability to analyse multi-sensory data, which translates into a higher degree of accuracy in the phase of recognition. Since one of the most favorable advantages of deep learning technology is the impressive power of automatic feature learning, using a neural network allows one to realize this goal. Convolutional Neural Network (CNN), which represents a branch of the Deep Neural Network (DNN), has provided excellent results for complex image classification tasks [14].

Data collected and labelled for localization of each node is an essential step for the training of the CNN. The data processed for each physical node location to be read and evaluated by the convolutional neural network must be converted appropriately in pixel grayscale images maps [15]. The images are referred to each tag in each zone where the white indicates the node receives a very high signal at a particular time point while the black means the node gets a little signal at that time. When the tag is tracked in movement and a different area, the intensity of the signal reported on the map changes rapidly, the images obtained will be used for training and testing the $\mathrm{CNN}$.

Convolutional neural network architecture: Through computational models consisting of several processing layers, CNN can learn data representations at multiple abstraction levels and represent a suitable solution to recognize the input images efficiently. CNN is a multiple structure network composed of several convolutional layers [15]. Each processing layer comprises a convolutional filter, an activation function, a pooling function, and a fully connected layer. At the end of each processing step, an input is generated for the next layer. In the convolutional operations, the trained filter set is convolved with input images to extract the specific feature to create the feature map, which becomes the input for the next filter.

The design of a CNN network requires a training period followed by a test phase. During the training phase, the images are labelled and transferred to the subsequent layers to allow the structure to convert from the representation level of the original input to a higher level and more abstract representation to constitute the reference feature maps with which the output feature maps must be compared. Once the training and test phase of the network has been completed, we will proceed with determining the accuracy level of the survey.

Conceptual model of CNN: CNN is a model of deep learning and is constituted by a set of different layers that act as extractors of the features of the input images and a fully connected terminal network that acts as a classifier. It has proved to be an effective solution for image recognition. They are built to analyze images included within certain data sets and classify objects in images within them. Each layer comprises three levels: Convolution, ReLu, and Pooling

- Convolutional Level (CONV) is the main level of the network. Its objective is to identify patterns. They are multiple, designed to identify features present in the initial image. Each layer learns to extract specific features of the images placed at its entrance. Various layers in cascade combine the features of the previous layers with higher programmed extraction levels.

- Rectified Linear Unit (ReLu) Level is placed after the convolutional level and has the task of canceling negative values obtained in the previous levels.

- Pool level it allows to identify if the study characteristic is present in the previous level.

The pooling layer obtains images with a specific resolution at the input and returns the same number of images with reduced pixels. The result of the convolution operations is the production of feature maps obtained with the help of filters that are matrices containing valid values for finding specific characteristics in the input images.

At the end of the sequence of convolutional layers, there is then the fully connected level (F.C.) which aims to establish the identifying classes obtained in the previous levels according to a certain probability. Each class represents a possible answer that the system will most likely choose. During the recognition phase, the network performs a classification operation to identify which class the input image belongs to, identifying the one with the highest probability. The values of the filters are initially chosen randomly; they are subsequently improved at each iteration of the process during the training phase. We estimate that the model's predictions are plausible with the loss function. In practice, the discrepancy between real values and predicted values is measured. The error is subsequently processed using the stochastic gradient technique. It is a cyclical technique consisting of two phases:

* Forward propagation,

* Updating the gradient value.

After the propagation phase, the outputs are produced to determine the prediction error compared with the expected ones. This error is used to calculate the gradient of the loss function, which is then propagated backward in the network (backpropagation). The gradient descent algorithm uses these values to update the assigned weights to minimize the loss function value.

\section{Movement Detection}

For patients with a physical and cognitive deficit, their localization and monitoring of their movement reduce the risk of falling and incident. Some accelerometers respond to both acceleration due to gravity and acceleration due to body movement for movement detection. These devices are suitable for monitoring postural orientation and body movement. For example, we can use a single accelerometer worn on the waist or multiple bodies worn [16]. MEMS technology enables devices that can process and analyse acceleration data providing final classification to the central unit $[17,18]$. To know the position of a subject in real-time about an environment, we can use a PIR (Passive Infrared) sensor 
that detects a warm object's movement like the people.

\section{ROUTING PROTOCOL}

The system's structure is composed of anchor nodes that are the fixed infrastructure arranged in the environment to monitor, by client nodes that the patients wear to support localization, tracking, and supervision of patients and control point for the management of the whole system. The data collected must be sent by nodes to the control point through a routing protocol that creates and controls the entire network's traffic. The choice of routing protocol is a key element in the design of Wireless Sensor Network. It must suffer several network limitations like bandwidth, energy, storage, and process of the central unit. There are different protocols:

a) mode of functioning,

b) participation style of the nodes,

c) network structure.

In the mode of functioning, we can distinguish:

- proactive protocol in which sensor node and transmitter sense the environment and send the data acquired via the predefined route to a Base station [19].

-reactive protocol in which if there are sudden changes over some predefined threshold value in the sensed attribute, the nodes immediately react, sending critical data to the user [20].

-hybrid protocol incorporates proactive and reactive protocols; their function is to calculate all routes available, and improvement is made during routing [21].

In the participation style of the nodes, we can distinguish:

-flat protocol in which each node that needs to transmit data first researches a valid route which is the shortest path to the Base station, and then sends.

-clustering protocol in which total area is divided into many clusters, each node in the cluster transmits its data to the corresponding cluster head (C.H.), which is in direct communication with Base Station.

-direct communication protocol in which each node can send direct information to Base Station

In the network structure, we can distinguish:

-hierarchical protocol is a routing used to perform energyefficient routing. There are two types of nodes: high energy nodes that process and send information; low energy nodes to sense in the interest area.

-data-centric in which the base station sends queries to a particular region of nodes and waits until their reply. Sensors of interested areas collect and sense data to the base station. This deletes redundancy in transmission.

-location-based control where information on the location of sensor nodes is needed. We can obtain location information from GPS signals, radio signal strength [22].

\section{METHODS AND TECHNICAL ROUTE}

We can develop the project in the following steps:

a. Study on the results achieved from previous investigations on the techniques used to monitor patients.

b. Analysis of sensors suitable for the localization, patient tracking, and monitoring biochemical parameters.

c. Identification of algorithms for determining locations and monitoring of subjects.

d. Integration and testing of HW and SW components.

e. Manufacturing of the prototype.

f. System test of data, collection of data, and presentation of results obtained.

\section{PROJECT SIGNIFICANCE}

To provide healthcare, a technological solution based on sensor and wireless communications system gives more effective treatment, safeguards sensitive patients, and reduces the cost of health expenditure. The solution that provides tags for patients and monitoring of their health state in-home allows the staff to intervene suddenly in critical cases reducing the need to resort to hospitalizations. For those already hosted in healthcare structures accelerates the pace and efficiency of treatment, reducing their stay time. Then with Information Technology, it's possible to enhance healthcare service quality and improve the entire operation timeless and efficiency through supporting activities such as tracking, monitoring, alerting patients and medical staff on demand.

\section{REQUIREMENTS OF THE PROPOSED SOLUTION}

The localization and tracking system proposed for healthcare has to meet the following requirement:

1) Accuracy in the localization of mobile subjects.

2) Scalability in the number of monitoring subjects and area to control.

3) Integration of sensor nodes of the different manufacturers.

4) Integrating and Interoperating with Healthcare Information Systems (HIS) to retrieve patients' information about care.

5) Devices must work optimizing energy consumption, ensuring a good level of operativity.

6) Devices will be chosen according to the cost, size, energy power, weight, comfortable for system users.

7) Fault Tolerance for single component and whole network.

\section{BRIEF BACKGROUND}

There are many systems for localization and tracking people with different HW/SW localization architecture. Below, the most common are grouped in four classes according to the principle used to create a reference database. The first systems collect the main parameters of known location and the storage in a database. The mobile objects are localized by matching their parameters and stored in the database.

a. Radar [23] is a radio-frequency system for locating and tracking people in an indoor environment. It works by processing signal intensity information at different base stations to provide overlapping coverage in the area of interest. To give people location, it merges measurements with the signal propagation model. RADAR 
uses signal intensity information gathered at multiple receiver locations to triangulate the patient's coordinates.

b. Landmark [24] is a localization system that enhances accuracy by using RFID tags and RFID readers to locate patients. It requires a further location tag by comparing the RSS of the nodes with those of reference tags. The RFID readers send the tags information to a central server with readers' I.D. and power level.

c. E.Z. [25] doesn't require the knowledge of the R.F. system or transmit power of anchor nodes. Instead, it depends on three elements: 1) the anchor nodes are enough to cover the indoor environment, 2) the user uses a smartphone or notebook 3) the mobile devices achieve location by GPS. Therefore, the mobile device needs to report the RSS corresponding to anchor nodes in their range at many locations to a central server. Finally, the server uses an algorithm to determine the location of the anchor node and their transmission power, then learns the characteristics of radiofrequency propagation to localize the users.

The second is based on the principle of localization of mobile objects by computing their distance from three or more anchor nodes using the measuring techniques and then applying one of the positioning techniques.

d. Cricket [26] is based on the recognition location rather than a location-tracking system. It identifies the location of mobile and anchor nodes by analysing information generated by the anchor node. Each anchor generates two signals: R.F. and ultrasound (U.S.) employed to locate the mobile node through the application of TDOA to determine their distance from anchor nodes. Then an inference algorithm is applied to determine the area in which the node is located.

e. Pinpoint [27] is based on location-tracking for indoor and outdoor environments. It uses the technique of TOA and is based on the exchange of messages between mobile nodes, storing their timestamps of sending and receiving to calculate the distance between a node and its neighbours. It is arranged in two steps, of which, in the first, each node estimates the distance to all its neighbours; in the second, combining all steps, the entire topology of the network is estimated.

f. Snowbat [28] is a system based on ultrasonic for tracking mobile objects with the TDOA measuring technique. This system's mobile objects are equipped with a sensor carrying an ultrasonic transmitter. At the same time, anchor nodes are provided of an ultrasonic receiver and radio transceivers positioned on both nodes for time synchronization and data transmission. The position of the mobile object is determined with the multi-lateration technique.

The third group uses a dense grid of anchor nodes whose position is known; the mobile object's position is considered the position of the nearest anchor node.

g. Traceme [29] is an indoor localization and tracking system with RFID technology. The system uses RFID active tags, RFID readers, the central server, and a database to store RFID data. Once the transmitter receives the data, the RFID readers send such data to the server that provides to update the database with information about the reader, transmitter, and relative area. There are two algorithms for the localization of mobile objects: one is based on short-range communication between tag and reader, the second is based on triangulation with RSSI between a tag and multiple readers in long-range communication. h. Will [30] uses smartphones and accelerometers, structured in two phases: training and service. During the training, the signal of anchor nodes and accelerometer are collected on the smartphones. These data are used to design, with data mining techniques, virtual rooms which are mapped to physical ones. During the service, the user queries the system sending its data. The search engine localizes the virtual space in which the patient is and, through the match between the physical room and virtual room, identifies the exact location.

The fourth group is based on the light-sensitive sensor to measure either distance or angle of the object to be located.

i. Flight [31] is a system of localization and tracking of a device-free object using light sensors. Each sensor is equipped with one light sensor and one transceiver to communicate, forming a connected WSN. For object tracking, the system uses a probabilistic method. The monitored area is divided into cells, to each cell is assigned a probability. The cell with the highest probability is associated with the moving object's position.

For the sensors employed, we report some experience done:

\section{BLOOD PRESSURE MONITORING}

Different solutions for cuff-less B.P. measurement have been proposed. The most common method is Pulse Transit Time (PTT) [32]; it is the interval between the peak of the R-wave in electrocardiogram (ECG) and the fingertip photoplethysmogram (PPG). It is related to arterial stiffness and can estimate systolic blood pressure (SBP) and diastolic blood pressure (DBP). Another method used is the ultrasound that measures the arterial diameter waveform along with the local PTT (in the form of pulse wave velocity) and then applies the Bramwell-Hill equation to compute the absolute pulse pressure (systolic BP-diastolic BP) [33]. Another method used is arterial tonometry is a long-standing method [34]. In theory, this method can measure a B.P. waveform without using a cuff by pressing a force sensor on an artery. The sensor must flatten or applanate the artery so that its wall tension is perpendicular to the probe. However, manual and automatic applanation has proven difficult, so the measured waveform is routinely calibrated with cuff B.P. values in practice. Therefore, the measurement acquired with this method requires calibration with cuff B.P. values.

The oscillometric finger-pressing can be an alternative to the previous methods [35]. It is a cuff-less B.P. measurement method. It can measure, via hydrostatic effect, systolic and diastolic B.P. without any calibration. These authors have used a ring embedded with PPG and a force sensor to measure the resulting variableamplitude blood volume oscillations and pressure applied on the finger. The measurements are strongly affected by the perfect alignment of the hand to the heart's height and the presence of motion artifacts that appear when the hand is moved rapidly or very slowly or for muscle contraction.

Chandrashaker et al. [36] have used a smartphone-based oscillometric finger-pressing method. The user serves as the actuator by pressing their finger against the phone to steadily increase the external pressure of the underlying artery (transverse palmar arch artery). In contrast, the phone is used as a sensor, rather than the cuff, to measure the resulting variable-amplitude blood volume variations or oscillations and applied pressure. On the back of the smartphone, a 3D-printed case is affixed. It's equipped with a photoplethysmography (PPG) sensor on the top of the force transducer to measure the blood volume oscillation and applied 
pressure. The smartphone runs an Android application to guide the finger actuation and a practical algorithm to compute systolic and diastolic B.P. at the brachial artery from the finger measurement. It is B.P. at the brachial artery rather than the finger that is the proven cardiovascular risk factor.

\section{Core Body Temperature Wireless Monitoring}

The measurement of core body temperature can be related to skin temperature, but few studies have been done on this topic. Niedermann et al. [37] have developed an algorithm to predict body temperature using skin temperature measured from the chest. However, the equipment highly professional was not suitable for continuous monitoring and long term. Lately, Woo et al. [38] have used a patch-type device that put on the skin over the clavicle measured humidity and skin temperature from which they predicted the body temperature. With an algorithm, they studied the relationship between perspiration rate and skin temperature to predict the body temperature; the error committed makes the method unreliable. Wei et al. [39] instead have used a measurement system of body temperature based on a wireless semiconductor sensor. The device is placed on the back under the neck because there is a thin layer of fat and muscle, ensuring skin temperature measurement close to the body temperature. The wireless health monitoring system comprises two parts: a wireless temperature measuring device attached to the back of the body and a receiver device to acquire data and send it to a computer for display and recording. The sensor used is a semiconductor for micro temperature with low power consumption and small measurement error equipped with an ARM microcontroller (Acorn- Reduced Instruction Set Computing Machine). The data measured are transmitted to a receiver using a multi-channel 2,4 GHz Gaussian frequency-shift keying (GFSK). The technology used enables the receiver to simultaneously communicate with a maximum of 128 transmitters. An algorithm based on the curve fitting method estimates the core body temperature based on skin temperature.

\section{Pyroelectric Infrared (PIR) Sensor}

PIR sensors are widely used for human tracking systems and localization due to the low cost and power consumption. In particular, an array of PIR sensors with digital output and equipped with Fresnel lens can track human motion by identifying walking people and entering or leaving the entrance of a building or room. Gopinathan et al. [40] using four PIR detectors, developed a motion tracking system based on coded apertures, which could detect human motion in one of the 15 cells in a $1.6 \mathrm{~m}$ square area. Shankar et al. developed a human tracking system using a sensor cluster consisting of PIR sensors and Fresnel lens arrays to implement the spatial segmentation [41]. Hao et al. [42] presented a human tracking system using an MSP430 family microcontroller, an R.F. transceiver, and a radial sensor module with eight PIR detectors with Fresnel lens arrays arranged around a circle. In addition, the system can track a single human target by detecting its angular displacement while moving.

Indeed, Jaeseok et al. [43] presented a study of human movement detection and localization using a PIR detector having two pairs of orthogonally aligned PIR sensors. They developed a data collection module consisting of two pairs of PIR sensors whose orthogonally aligned dual sensing elements. Fresnel lenses were modified to narrow the field of view of the PIR sensors to its horizontal motion plane, a data logger, amplifier circuits, and a rechargeable battery.

They set three PIR detectors in a room for localizing people; one
PIR sensor was placed on the ceiling; two PIR sensors were placed on opposite walls facing each other. A data set was collected from eight subjects walking in three different conditions: two directions (back and forth), three distance intervals (close to one wall sensor, in the middle, close to the other wall sensor), and three-speed levels (slow, moderate, fast). They employed two feature sets: a raw data set and a reduced feature set composed of amplitude and time to peaks; and passage duration extracted from each PIR sensor. In addition, they performed classification analysis considering different configurations such as:

-the number of detectors involved (ceiling-mounted detector vs. wall-mounted detector),

-the number of PIR sensors involved (a single PIR sensor, a pair of PIR sensors orthogonally aligned and two pairs of PIR sensors orthogonally aligned),

-the feature set (raw data set vs. reduced feature set) and machine learning algorithms such as support vector machine (SVM) and Bayes net.

The movement detector device comprises two PIR sensors, a data logger, a rechargeable battery, and a custom-fabricated data acquisition board. PIR detectors are separated by $2 \mathrm{~cm}$ of distance, including the Fresnel lens. Since PIR sensors generate a low voltage signal, they integrate op-amplifier circuits to amplify the low PIR output signal. To acquire amplified PIR sensor signals, they created a printed circuit board (PCB) having PIR sensors and op-amp circuits, whose outputs are connected to the analog inputs of a data logger.

\section{Mental Stress Monitoring}

An innovative method to monitor mental stress, compared to the traditional one that uses ECG signal through an extraction process of long-term heart rate variability (HRV), is a deep learning network that uses an ultra-short-term raw ECG signal (Deep ECGNet) [44].

It's composed of recurrent (RNN) and convolutional neural networks (CNN) architecture with optimal convolutional filter length and pooling length. The results have been achieved through experiments and analysis on the waveform characteristics of ECG signals. According to various experimental protocols to elicit stressful conditions, the data have been obtained by two groups of people, one in Korea and the other in Belgium. In addition, psychophysiological studies have demonstrated that physiological features are associated with cardiovascular disorders. Belkic et al. [45] have also shown that psychological stress is a risk factor for cardiovascular disease. ECG, recorded from the electrophysiological pattern of heart muscle during a heartbeat, can be a biomarker for mental stress status. Previously interviews and questionnaires were used to study the stress; subsequently, other protocols such as biochemical samples, physiological signal, EMG, EEG, blood pressure have been developed.

The beat-to-beat alternations, heart rate variability (HRV) have been used to monitor the mental stress status [46]. The longterm ECG recording to calculate HRV has been replaced with ultrashort recording. A new methodology has been used to study the ultra-short ECG recordings with the help of novel deep learning architecture. The researchers demonstrated that this methodology was effective in image recognition due to its automatic extraction of data characteristics using convolution and pooling operation. 
In this case, convolutional neural networks (CNN) and recurrent neural networks (RNN) have extracted features from ECG signals to replace conventional HRV parameters.

The solution adopted, called the DeepECG model, comprises a single convolution filter, ReLu function, single pooling layer, and dropout. Before the classification, SoftMax two-layer alternatively of batch processing and RNN have been added. The batch processing was performed in mini-batch units, known to prevent exploding/vanishing of parameters when gradient descent is used. RNNs were added to extract the final feature using the extracted sequential features from the CNNs' stage. This architecture has been determined by comparing its performance with those of the other two structures of Deep Learning; one is done by setting up an empirical model that, unlike the first, had three convolution layers and three pooling layers associated with three dropouts. Dropout is a regularization technique to prevent the overfitting problem of the neural network on training data. The second architecture is a general CNN model that, compared with the first, has two fully connected layers before the batch processing and lacks the RNN layer. Therefore, the RNN model is necessary to preserve the sequential order of the ECG signal. The Deep ECGNet requires fewer hardware resources and fast execution time than other models. The neural network requires a training phase on an entire process from the prior knowledge of signal input of waveforms and intervals of ECG. Different experiments have determined the optimal convolution filter length and pooling length by which it's possible to extract key features of a one-cycle ECG signal based on the specific duration. Compared with conventional stress analysis methods (HRV and Spectrogram), the performance is higher.

\section{REFERENCES}

1. Aravind KG, Chakravarty T, Chandra MG, Balamuralidhar P (2009) On the architecture of vehicle tracking system using wireless sensor devices. 2009 International Conference on Ultra-Modern Telecommunications \& Workshops, pp. 1-5.

2. Ren Y, Pazzi R, Boukerche A (2010) Monitoring patients via a secure and mobile healthcare system. Wireless Communications IEEE 17(1): 59-65.

3. Bharathidasan A, Anand V (2002) Sensor networks. Technical Report, Dept of Computer Science, University of California, Davis, USA

4. Yick J, Mukherjee B, Ghosal D (2008) Wireless sensor network survey. Computer Networks 52(12): 2292-2330.

5. Patwari N, Ash J, Kyperountas S, Hero I, Moses R, et al. (2005) Locating the nodes: Cooperative localization in wireless sensor networks. Signal Processing Magazine IEEE 22(4): 54-69.

6. Mao G, Fidan B, Anderson BD (2007) Wireless sensor network localization techniques. Computer Networks 51(10): 2529-2553.

7. Ko J, Gao T, Rothman R, Terzis A (2010) Wireless sensing systems in clinical environments: Improving the efficiency of the patient monitoring process. Engineering in Medicine and Biology Magazine IEEE 29(2): 103-109.

8. Wang J, Ghosh R, Das S (2010) A survey on sensor localization. Journal of Control Theory and Applications 8: 2-11.

9. Liu H, Darabi H, Banerjee P, Liu J (2007) Survey of wireless indoor positioning techniques and systems. Systems, Man, and Cybernetics, Part C: Applications and Reviews IEEE Transactions 37(6): 1067-1080.

10.Zhu Y, Huang D, Jiang A (2008) Network localization using angle of arrival. Electro/Information Technology, 2008. EIT 2008. IEEE International Conference, pp. 205-210.

11. Altini M, Brunelli D, Farella E, Benini L (2010) Bluetooth indoor localization with multiple neural networks. In: Wireless Pervasive Computing (ISWPC). $5^{\text {th }}$ IEEE International Symposium, pp. 295-300.
12. Feldmann S, Kyamakya K, Zapater A, Lue Z (2003) An indoor bluetoothbased positioning system: Concept, implementation and experimental evaluation. International Conference on Wireless Networks, p. 272.

13. Madhavapeddy A, Tse A (2005) A study of Bluetooth propagation using accurate indoor location mapping. Ubi-Comp 2005: Ubiquitous Computing, pp.105-122.

14. Bibbò L, Carotenuto R (2021) An integrated system for indoor people localization, tracking, and monitoring. Journal of International Scientific Publications, Materials, Methods \& Technologies, Vol. 15.

15. LeCun Y, Bengio Y, Hinton G (2015) Deep learning. Nature 521: 436 444 .

16. Mathie M, Coster A, Celler B, Lovell N (2004) Classification of basic daily movements using a triaxial accelerometer. Medical and Biological Engineering and Computing 42(5): 679-687.

17. Koen Van C (2012) Modeling RF MEMS devices. IEEE Microwave Magazine 13(1): 83-110.

18. Polla L (2001) BioMEMS applications in Medicine. IEEE International Symposium on Micromechatronics and Human Science, pp. 13-15.

19. Katiyar V, Chand N, Gautam GC, Kumar A (2011) Improvement in LEACH protocol for large-scale wireless sensor networks. 2011 International Conference on Emerging Trends in Electrical and Computer Technology (CEITEC), India.

20. Prasanna S, Srinivasa R (2012) An overview of wireless sensor networks applications and security. In: International Journal of Soft Computing and Engineering (IJSCE) 2(2): 2231-2307.

21. Parul T, Surbhi J (2012) Comparative study of routing protocols in wireless sensor network. International Journal of Advanced Research in Computer Science and Software Engineering 2(9).

22. Shio KS, Singh MP, Singh DK (2010) Routing protocols in wireless sensor networks a survey. International Journal of Computer Science \& Engineering Survey (IJCSES) 1(2).

23. Bahl P, Padmanabhan V (2000) Radar: an in-building rf-based user location and tracking system. INFOCOM 2000: Nineteenth Annual Joint Conference of the IEEE Computer and Communications Societies, Proceedings IEEE 2: 775-784.

24. Ni L, Liu Y, Lau YC, Patil A (2003) Landmarc: Indoor location sensing using active RFID. Pervasive Computing and Communications, 2003. Proceedings of the First IEEE International Conference, pp. 407-415.

25. Chintalapudi K, Padmanabha IA, Padmanabhan VN (2010) Indoor localization without the pain. Proceedings of the Sixteenth Annual International Conference on Mobile Computing and Networking, ser. MobiCom '10, New York, USA, pp. 173-184.

26. Priyantha NB, Chakraborty A, Balakrishnan H (2000) The cricket location-support system. Proceedings of the $6^{\text {th }}$ annual international conference on Mobile computing and networking, ser. MobiCom '00. New York, USA, pp. 32-43

27. Youssef M, Shankar U (2006) Pinpoint: An asynchronous time-based location determination system. Fourth International Conference on Mobile Systems, Applications, and Services (MobiSys 2006), pp. 165176.

28. Baunach M, Kolla R, Mühlberger C (2007) SNoW Bat: A high precise WSN based location system. Institut für Informatik, University of Wuerzburg, Germany, p. 424.

29. Silva P, Paralta M, Caldeirinha R, Rodrigues J, Serodio C (2009) Traceme x2014: Indoor real-time location system. $200935^{\text {th }}$ Annual Conference of IEEE Industrial Electronics, pp. 2721-2725.

30. Wu C, Yang Z, Liu Y, Xi W (2012) Will: Wireless indoor localization without site survey. Parallel and Distributed Systems, IEEE Transactions, 10(10): 1-11.

31. Mao X, Tang S, Xu X, Li XY, Ma H (2011) iLight: Indoor device-free passive tracking using wireless sensor networks. 2011 Proceedings IEEE INFOCOM, pp. 281-285. 
32. Arza A, Lázaro J, Gil E, Laguna P, Aguiló J, et al. (2013) Pulse transit time and pulse width as potential measure for estimating beat-to-beat systolic and diastolic blood pressure. Computing in Cardiology 40: 887890.

33. Seo J, Pietrangelo SJ, Lee HS, Sodini CG (2015) Non-invasive arterial blood pressure waveform monitoring using two-element ultrasound system. IEEE Trans Ultrason Ferroelectr Freq Control 62: 776-784.

34. Hansen S, Staber M (2006) Oscillometric blood pressure measurement used for calibration of the arterial tonometry method contributes significantly to error. Eur J Anaesthesia 23(9): 781-787.

35. Shaltis PA, Reisner AT, Asada HH (2008) Cuffless blood pressure monitoring using hydrostatic pressure changes. IEEE Trans Biomed Eng 55(6): 1775-1777.

36. Chandrasekhar A, Kim CS, Natarajan NK, Hahn JO, Mukkamala R (2018) Smartphone-based blood pressure monitoring via the oscillometric finger-pressing method. Sci Transl Med 10(431): eaap8674.

37. Niedermann R, Wyss E, Annaheim S, Psikuta A, Davey S, et al. (2013) Prediction of human core body temperature using non-invasive measurement methods. International Journal of Biometeorology 58(1): 7-15.

38. Woo SH, Din ZM, Lee JW (2009) Body temperature predicting patch-type telemedicine system. IEICE Electronics Express 6(16): 1161-116.

39. Wei Q, Park HJ, Hyun LJ (2019) Development of a wireless health monitoring system for measuring core body temperature from the back of the body. Journal of Healthcare Engineering 2019: 8936121.
40. Gopinathan U, Brady DJ, Pitsianis NP (2003) Coded apertures for efficient pyroelectric motion tracking. Opt Express 11(18): 2142-2152.

41. Shankar M, Burchett JB, Hao Q, Guenther BD, Brady DJ (2006) Humantracking systems using pyroelectric infrared detectors. Opt Eng 45: 106401.

42. Hao Q Brady DJ, Guenther BD, Burchett JB, Shankar M, et al. (2006) Human tracking with wireless distributed pyroelectric sensors. IEEE Sens J 6(6): 1683-1696.

43. Jaeseok Y, Sang-Shin L (2014) Human movement detection and identification using pyroelectric infrared sensors. Sensors 14: $8057-$ 8081.

44. Hwang B, You J, Vaessen T, Germeys I, Park C, et al. (2018) Deep ECGNet: An optimal deep learning framework for monitoring mental stress using ultra short-term ECG signals. Telemedicine and E-Health 24(10): 753772 .

45. Belkic K, Landsbergis P, Schnall PR, Baker D, Theorell T, et al. (2000) Research findings linking workplace factors for CVD outcomes: Psychosocial factors- Review of the empirical data among men. Occup Med 15: 24-46.

46. Task Force of the European Society of Cardiology and the North American Society of Pacing and Electrophysiology. (1996) Heart rate variability: Standards of measurement, physiological interpretation, and clinical use. Eur Heart J 93(5): 1043-1065. 